\title{
Several conjectures about the existence of the ether
}

\author{
JinjunCheng; DianCheng
}

In November 2021

[ abstract ] The "zero result" of Michelson Morley's experiment was not enough to deny the existence of a fifth element---ether, as conceived by Aristotle. Hubble's explanation of the Doppler effect of the systematic cosmic the red shift was incomplete and needed to be introduced into the ether.The physical significance of universal gravitation constant reveals the possibility of the existence of ether. After much thought and logical reasoning, there is good reason to think that the ether is negative energy, stream of consciousness, and space itself, it combines with photons to build elementary particles, elementary elements, everything in the universe, including ourselves. In addition, it is feasible to explain the causes of gravitation, electromagnetic force, strong force and weak force from the perspective of the ether, which seems to be easier for us to understand. The most tragic conclusion is that the universe was created out of nothing and will eventually return to nothing.

[ Key words ] ether negative energy stream of consciousness space 
According to The Ancient Greek philosopher Aristotle, the world was made up of water, fire, air and earth, as well as ether, a fifth element high in the sky. The ether is pure, transparent, perfect, and immutable, the sun, moon, planets, stars and other celestial bodies are made of ether.In the history of science, the ether started out as a kind of mystery. Later, it gradually became a kind of material concept assumed by physicists in certain historical periods. The 17th century philosopher Descartes, who introduced the ether to science and ascribed its mechanical properties, believed that all forces between bodies were transmitted through the ether.Huygens developed Hooke's theory of light waves and believed that the medium of light waves was ether. Newton's laws of gravity seemed to support the action of space at a distance, but he himself preferred the existence of the ether, which he believed could transmit vibrations, but not light waves. To sum up, physicists conceptualize the ether as a background medium throughout the universe that transmits light and force. Because physicists believe the ether can transmit force, the movement of the earth in the ether creates an "ether wind", bending light waves as if sound waves were deflected in a stream. In 1887, Michelson and Morley designed their famous experiment in search of the ether, along these lines, but found no "ether wind" and no difference in the speed of light in any direction, upending all theories about the existence of the ether. Although many physicists tried to save the ether until the early 20th century, after the establishment of special 
relativity, Faraday, Maxwell, and Lorenz's theory of electromagnetic fields was considered to be a form of matter itself, propagating as waves in a vacuum without the need for ether at all.In this way, the field theory replaced the ether theory, which left only conceptual bodies and unyielding souls wandering aimlessly and alone in the world.

Does the ether really exist? Does the creator leave everything to be scattered in the universe? Could there be an ether? Is it the dwelling place of all things? Is it a boundless, eternal existence? How does it relate to everything in the world? How can it transmit gravity without creating an "ether wind" that interferes with the direction of light? It's really exciting that there are so many questions about the ether that need to be addressed. But to save the ether, the Michelson Morley experiment must be answered scientifically.

\section{The Michelson Morley experiment was not enough to deny the existence of the ether}

Since great scientists such as Newton, Maxwell, and Lorenz were convinced that the ether existed, experimental physicists kept trying to find it. In 1728, The British astronomer Bradley, after analyzing the phenomenon of aberration of stars, believed that the speed of the earth in the ether is the speed of the revolution around the sun, if the speed of light is measured on earth, it should be measured differently in different directions because of the influence of the "ether wind" caused by the earth's revolution. In 1887, American scientists Michelson and Morley designed the experiment, is to try to find evidence for 
the existence of the ether by discovering this difference in the speed of light.

\subsection{Introduction to the Michelson Morley experiment}

Michelson designed the interferometer (see picture below) with a few basic building blocks: On the left is a light source, labeled S; The beam reaches a central point with a half-silvered mirror, half of which is reflected vertically and the other half straight through; The light is then split into two beams, directed in their respective directions $\mathrm{M}_{1}$ and $\mathrm{M}_{2}$; The two beams of light reach their respective mirrors and then bounce back to the center. Since arm lengths $\mathrm{L}_{1}$ and $\mathrm{L}_{2}$ are equal, the time required for a round trip should also be equal. If the speed of the beam is $\mathrm{C}$ and each beam travels in one direction for a distance of $\mathrm{L}$, the time required for a round trip is $2 \mathrm{~L} / \mathrm{C}$. Since $\mathrm{L}_{1}=\mathrm{L}_{2}$, when the two beams of light return to the center of the interferometer, the peak of one beam and the peak of the other beam can completely overlap, and the trough can also completely overlap, without interference. However, if an "ether wind" is blowing in the opposite direction ( $\mathrm{v}$ is $30 \mathrm{~km} / \mathrm{s}$, which is equal to the speed of the earth's revolution), the two perpendicular beams of light will travel a different distance and take a different time to pass through the two long arms, and you should see the interference stripes as they meet. However, the Michelson Morley experiment did not find any interference between the two beams, the speed of the "ether wind" was zero, and there was no displacement between the earth and the ether. 


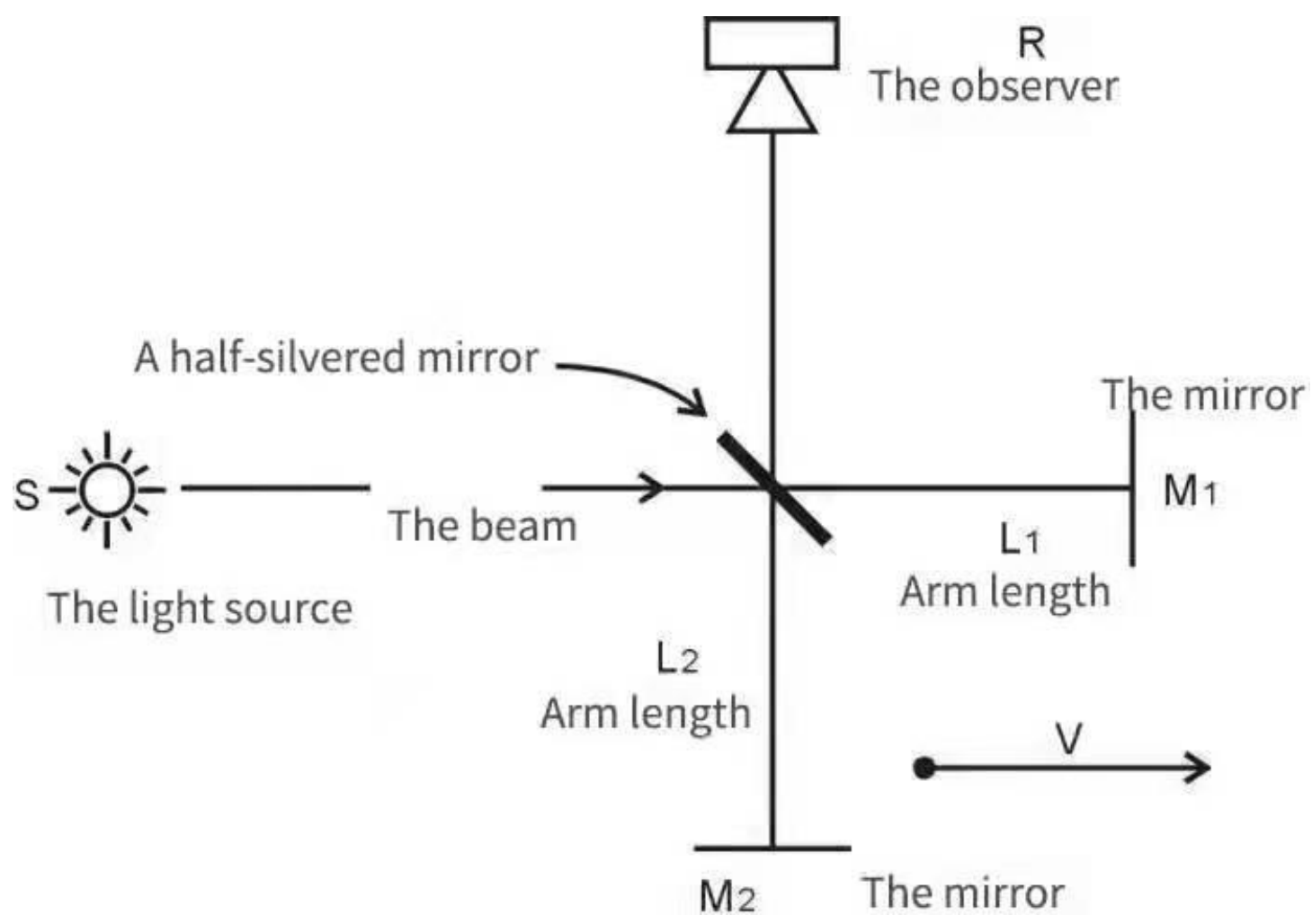

The Michelson Morley experiment is an excellent example of precision, ingenuity and repeatability (details of the process and data can be found in the literature, which will not take up space here). If the ether is static with respect to the sun, how can it be static with respect to the earth? To solve this intractable difficulty, Einstein discarded the ether and developed his famous special theory of relativity, he argued that the ether was not needed for the propagation of light, and that the speed of light $\mathrm{C}$ was constant in any frame of reference.

\subsection{Interpretation of the Michelson Morley experiment}

Michelson Morley's ideas were correct, but his conclusions were incomplete. Suppose that in the context of space without the ether, because the observer of this experiment is in the Earth's inertial system, the distance between the vertical direction and the horizontal direction is the same, and the speed of light $\mathrm{C}$ is constant, the observation result must be two beams of light arriving at the 
same time, without phase difference (as if observing in a uniformly moving train). But an observer in the sun's inertial frame would notice that the two beams do not arrive at the same time, resulting in a phase difference, this is what special relativity calls simultaneity relative.

Let's say that in the context of ether, since the experiment was conducted from earth, could an observer in the earth's inertial frame detect an "ether wind"? Although the idea and method of the experiment are correct, the preconditions of the experiment are as follows: as a space medium, ether has mechanical physical properties and can collide with light mechanically. If the ether had the physical properties of air, that is, made of elemental particles and had mass, then the "ether wind" would have to collide with two perpendicular beams of light, among them: the light perpendicular to the direction of the earth's movement is bound to deviate, the speed of light remains unchanged, but the back and forth optical path becomes longer; The speed of light parallel to the earth's motion direction is not affected, and the return path of light is unchanged. Therefore, from the perspective of the earth observer, two beams of light will have phase difference and cannot arrive at the same time. As the "ether wind" velocity was measured at 0 , no evidence of ether was found. One explanation for the results of this experiment is to discard the ether completely, as Einstein did, denying its existence; however, there is another possible explanation, that is, ether, as the background medium of space, has the property different from mass material, and does not have mechanical interaction with moving light 
under certain conditions, that is, the interaction force is 0 . If this were true of the properties of the ether, it would not prevent it from continuing to exist as a spatial background medium (see below for details).

In this way, ether was temporarily rescued from the "near-death state" and began to have some signs of life, but whether it can really come back to life depends on the success of further theoretical reasoning.

\section{A complete explanation of Hubble's redshift necessitates the}

\section{introduction of the ether}

In 1929, The American astronomer Hubble found through analyzing the red frequency shift of the wavelengths of extragalactic galaxies that these galaxies have systematic red shift phenomenon, and the amount of red shift $Z=\left(\lambda-\lambda_{0}\right)$ $/ \lambda_{0}\left(\lambda_{0}\right.$ and $\lambda$ are the wavelengths before and after the red shift respectively) is proportional to the distance $d$ from the galaxy to our observation point, that is, $\mathrm{Z}=\mathrm{KD}$ ( $\mathrm{K}$ is a proportionality constant), this systematic redshift of galaxies is called the Hubble redshift. As for the cause of galaxy redshift, Hubble believed that it was caused by the Doppler effect of galaxy light wave, thus deduced the relationship between the receding velocity $\mathrm{V}$ of the measured galaxy relative to us and the redshift quantity $\mathrm{Z}: \mathrm{V}=\mathrm{CZ}$ ( $\mathrm{C}$ is the speed of light), and further deduced the famous formula: $\mathrm{V}=\mathrm{H}_{0} \mathrm{D}$ (where $\mathrm{H}_{0}$ is called Hubble's constant, $\mathrm{V}$ is the radial velocity between galaxies, and $\mathrm{D}$ is the distance between galaxies), this is Hubble's law. Hubble further speculated that the red shift of the quasar spectrum was not caused by its own motion, but by the uniform expansion of 
space everywhere, which led to the big Bang theory.

\subsection{Hubble's law is not complete}

However, a number of recent astronomical observations show that the Hubble constant is not constant and the Hubble relation cannot be strictly established. At distances of less than 5 billion light-years, Hubble's constant accords with the measurements of a large number of extragalactic galaxies; but for more distant galaxies, the actual values are far from Hubble's constant, and the actual redshift is greater than the Doppler redshift, meaning that some of the energy of starlight from these distant galaxies somehow disappears, if the Doppler effect is used to explain the Hubble redshift phenomenon, the light will not obey the law of energy conservation in the process of motion. In addition, when the value of starlight redshift $Z$ is greater than 1 , it will also cause the predicament that the galactic apparent receding velocity $\mathrm{V}$ exceeds the speed of light C. Therefore, Hubble's law based on Doppler effect is not a complete explanation of Hubble redshift.

\subsection{Non-Doppler effect interpretation of Hubble redshift}

Let's go back to the origin of the event and re-analyze it. The relationship between the redshift and distance of the original galaxies observed by Hubble was $\mathrm{Z}=\mathrm{KD}$. Since Hubble believed that the galaxy redshift was caused by the Doppler effect, the relation $\mathrm{V}=\mathrm{CZ}$ was derived, and the Hubble relation $\mathrm{V}=\mathrm{H}_{0} \mathrm{D}$ was further derived. If you take the Doppler effect out of the equation and go back to the original observations, Since $Z=K D$ (Formula 1 ) is derived from the 
original observation, this formula is valid and $\mathrm{K}$ is a proportionality constant.

Since $\mathrm{D}=\mathrm{CT}, \mathrm{C}$ is the speed of light and $\mathrm{T}$ is the time it takes for starlight to travel from the galaxy to our observation point; Substituting $D=C T$ and $Z=(\lambda$ $\left.\lambda_{0}\right) / \lambda_{0}$ into formula 1 , we get $:\left(\lambda-\lambda_{0}\right) / \lambda_{0}=\mathrm{KCT}$ (formula 2); Because the photon energy formula $\mathrm{E}=\mathrm{h} \mathrm{V} \quad(\mathrm{h}$ is Planck constant, $\quad \mathrm{V}$ is the frequency of photon), and $\mathrm{C}=\lambda \mathrm{V}$; let $\mathrm{V}_{0}$ and $\mathrm{V}$ be the frequency before and after the red shift of starlight respectively, $\mathrm{E}_{0}$ and $\mathrm{E}$ are the energy before and after the red shift of starlight respectively, and substitute into Formula 2 to obtain: $\left(\mathrm{E}_{0^{-}} \mathrm{E}\right) / \mathrm{E}=\mathrm{KCT}$. If $\triangle \mathrm{E}=\mathrm{E}_{0^{-}} \mathrm{E}, \triangle \mathrm{E} / \mathrm{E}=\mathrm{KCT}$ (Formula 3).

Since $\mathrm{KC}$ is a proportionality constant, it can be seen that the energy $\triangle \mathrm{E}$ lost by starlight from the motion of the galaxy to our observation point is proportional to the time t of the motion. Because the speed of light $\mathrm{C}$ is constant, it takes longer for the light from the more distant galaxy to reach our point of view, thus, starlight from a distant galaxy beyond the river loses more energy $\triangle \mathrm{E}$ than nearby galaxies, and we observe a larger redshift for the galaxy.

Hubble observed that the relationship between the redshift of starlight and distance $\mathrm{Z}=\mathrm{KD}$ was an objective phenomenon, if this is not caused by the Doppler effect, it must be caused by the energy of the starlight decreasing over time as it moves towards us. We know that starlight is made up of a single photon, according to Planck's quantum hypothesis, the energy of each photon moving in space must gradually decay in a quantized way with the moving time, that is, a discontinuous portion of the energy radiates outwards. If a photon is 
moving in a void, then a fraction of the energy radiated outward by a photon must produce multiple photons, however, this phenomenon is inconsistent with actual observation and current optical theory; if a photon does not radiate outward to separate multiple photons, the photon's energy will not decay, and we would not be able to observe the galaxy redshift. Therefore, it is speculated that the space carrying the movement of photons cannot be a real void, and the space must be filled with some mysterious elements. Since photons radiate energy discontinuously as they move through space, and this energy seems to be absorbed by space, Presumably this mysterious space-filled element would have a negative energy characteristic that could be neutralized by the positive energy emitted by photons in the process, the energy of the photons that cause space to move decays over time. In this way, one photon does not split into multiple photons, we can also observe the redshift of starlight. Hence the inference: this negative energy that fills the space of the universe should be the ether, the fifth element predicted by Aristotle.

\section{The universal gravitational constant $G$ revealed the possibility of the}

\section{ether}

\subsection{Newton's law of gravitation}

Newton uses calculus thought after Kepler three laws are summarized, discovered the law of universal gravitation. In 1687, Newton proposed in "the mathematical principles of natural philosophy" gravitation size only related to the quality of the object and the distance, the $F=G_{1} M_{2} / R^{2}, G$ is the 
gravitational constant, $\mathrm{M}_{1}$ and $\mathrm{M}_{2}$ is the quality of the two objects, $\mathrm{R}$ is the distance between the two substances, this is the famous formula for gravity. In 1789, Cavendish measured the value of $\mathrm{G}$ by torsion balance experiment, and the formula of universal gravitation was perfected. But how does gravity work? Why do things attract each other? Newton explained this by saying: the universal law of gravitation is an inherent property of mass, at that time, it was believed that gravitation was a force that transcends distance and could be transmitted to an infinite distance in an instant without any action time.

\subsection{Einstein's equations of gravitational field}

In 1905, Einstein proposed in his special theory of relativity that "the speed of light in a vacuum is the ultimate speed of information", but this contradicts Newton's law of universal gravitation's idea of transcends distance. For this reason, in 1915 Einstein proposed the equations of the gravitational field in his general theory of relativity: Ruv- $0.5 \operatorname{Rguv}=8 \pi \mathrm{GT}_{\mathrm{UV}} / \mathrm{C}^{4}$ ( $\mathrm{G}$ is the universal gravitational constant), he also explains the nature of gravitation, and thinks that gravitation is not real, but an illusion, and its nature is caused by the distortion of space and time around by mass objects. For example, the iron ball is placed in the middle of the spring bed, and then a glass ball is thrown along the spring bed. The weight of the iron ball causes the spring bed to sag, so the glass ball will rotate around the iron ball several times and then fall to the iron ball. This phenomenon is essentially the same as the planets around the stars. Einstein's equations of gravitational field are of great significance because they reveal the 
relationship between the distribution of matter in space-time and the geometry of space-time (the former determines the latter).

Einstein's general theory of relativity did not overturn or replace Newton's theory of gravitation, but derived on the basis of the theory of gravitation. Einstein's equations of gravitational field have always used Newton's gravitational constant G. We know that Newton never explained the meaning of the gravitational constant $G$, as if $G$ were just a meaningless constant; the $G$ in Einstein's gravitational field equations came from Newton's laws of gravity, but he didn't say what $\mathrm{G}$ actually meant physically. Einstein summed up the cause of gravity by saying that matter tells space-time how to curve, space-time tells matter how to move, but without the background medium of etheric space, it is indeed difficult for human understanding to imagine how the void could be curved. Therefore, the key to reveal the nature of universal gravitation is to find the physical meaning of universal gravitation constant G.

\subsection{Physical significance analysis of gravitational constant}

3.3.1 Elastic shrinkage force of ether. It is assumed that ether exists as a space background medium, which is used to carry the existence and movement of mass objects and has elastic force. When there is no mass bearing object in etheric space, the etheric space is uniform and does not produce force; When a mass object $\mathrm{A}$ in the etheric space, it will cause elastic deformation of the etheric space around the mass object A, and produce elastic contraction force pointing to the center of gravity of $\mathrm{A}$, which is the same as the effect of placing 
an iron ball in the center of the elastic trampoline plane (see the following figure).

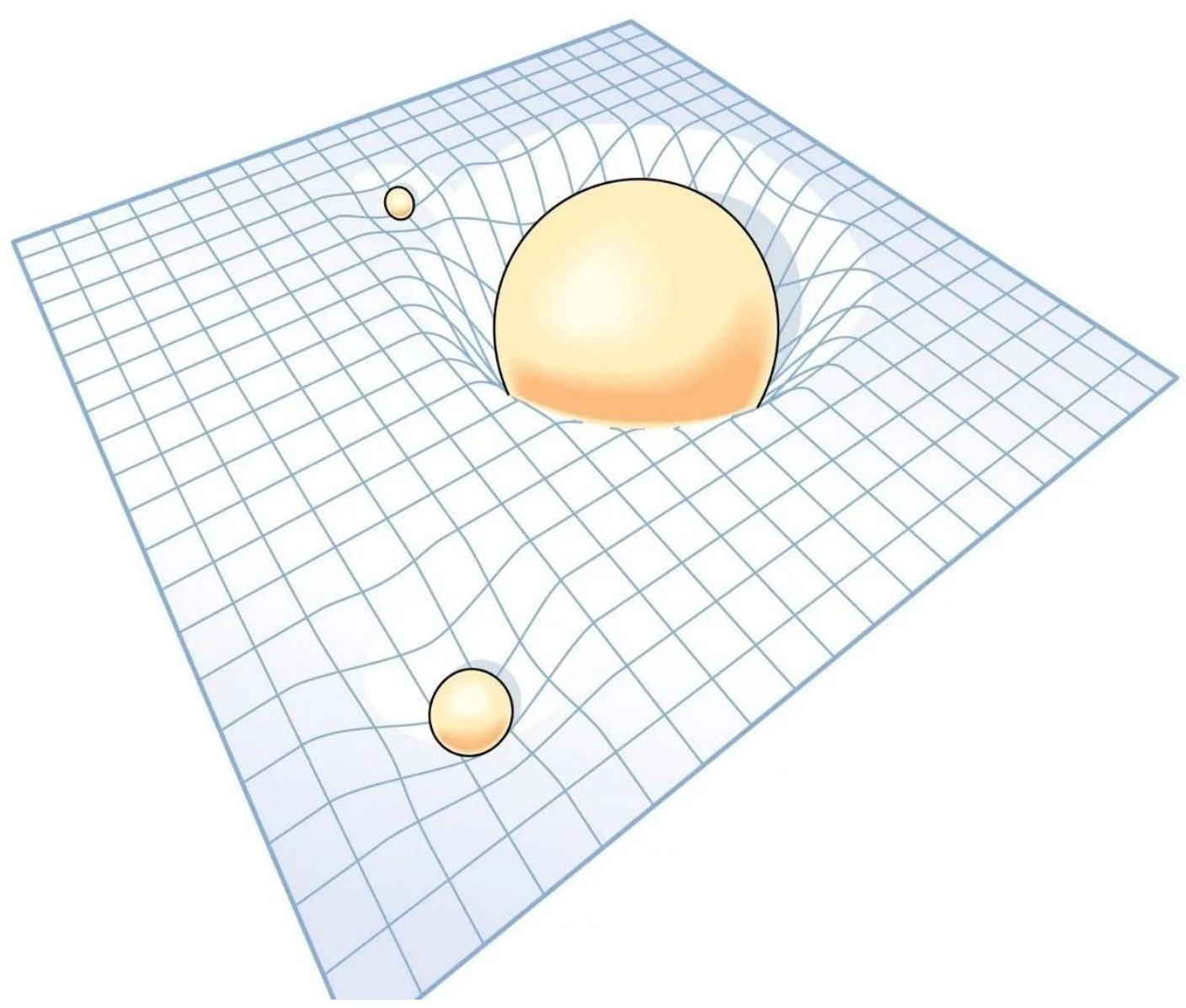

Mass object A causes the process of elastic deformation in the ether space, showing the characteristics of three-dimensional space: that is, with the spatial position of the center of gravity of A as the core, it spreads evenly to the surrounding space like sphere; as the deformation radius $\mathrm{R}$ continues to extend, the deformation diffusion to the corresponding spherical shell area $4 \pi R^{2}$ also increases. Most importantly, as the ether deformation continues to spread in space, forces are generated from various positions on the corresponding spherical shell surface to the space position of the center of gravity of the mass 
object A, this force is the elastic shrinkage force of the ether caused by A. The same as the principle of planar spring bed, the greater the mass of mass object A, the greater the etheric elastic contraction force; at the same time, the elastic shrinkage force induced by A gradually weakened in the conduction process from the center of gravity point of A to the outside.

If at this time, another mass object B is also in the etheric space, it is bound to be affected by the etheric elastic contraction force triggered by mass object A, that is, by the attraction of $\mathrm{A}$, in turn, $\mathrm{A}$ is affected by the etheric elastic contraction force exerted by $\mathrm{B}$, that is, by the attraction of $\mathrm{B}$. Therefore, it can be said that there is A mutual attraction between two mass objects $\mathrm{A}$ and $\mathrm{B}$ in the etheric space. The attraction is proportional to the mass of $\mathrm{A}$ and $\mathrm{B}$, and inversely proportional to the space distance from A to $\mathrm{B}$. Therefore, it is inferred that the elastic contraction force caused by the mass object in the Ether space should be the universal gravitation, and the universal gravitation constant $\mathrm{G}$ must be related to the elastic contraction force of the ether.

3.3.2 Universal gravitational constant $\mathbf{G}$ physical significance. We know that Newton's formula for universal gravitation is $F=\mathrm{GM}_{1} \mathrm{M}_{2} / \mathrm{R}^{2}$, the physical units of the universal gravitational constant $G$ are: $N \times \mathrm{m}^{2} / \mathrm{kg}^{2}$. Since $1 \mathrm{~N}=1 \mathrm{~kg} \times$ $\mathrm{m} / \mathrm{s}^{2}$, the units of $\mathrm{G}$ can be changed as: $\mathrm{m}^{3} /\left(\mathrm{kg} \times \mathrm{s}^{2}\right), \rightarrow\left(\mathrm{m}^{2} / \mathrm{s}^{2}\right) \times(\mathrm{m} / \mathrm{kg}) ; \rightarrow$ $\mathrm{v}^{2} \times(\mathrm{m} / \mathrm{kg}) ; \rightarrow\left(\mathrm{v}^{2} / \mathrm{m}\right) \times\left(\mathrm{m}^{2} / \mathrm{kg}\right)$, Where $" \mathrm{v}^{2} / \mathrm{m}^{\prime}$ is the unit of centripetal acceleration of circular motion. We analyze the physical meaning of the gravitational constant $\mathrm{G}$ by using the elastic contraction force in etheric space. 
For example: Suppose mass A and mass B are both in the ether, the mass of $\mathrm{A}$ is greater than the mass of $\mathrm{B}$, the gravitational attraction $\mathrm{F}$ between $\mathrm{A}$ and $\mathrm{B}$ attracts B to move uniformly in A circle around A (ideal space state), $v$ is the velocity of $\mathrm{B}$ along the tangent direction, and the distance from $\mathrm{A}$ to $\mathrm{B}$ is $\mathrm{R}$ (see figure below).

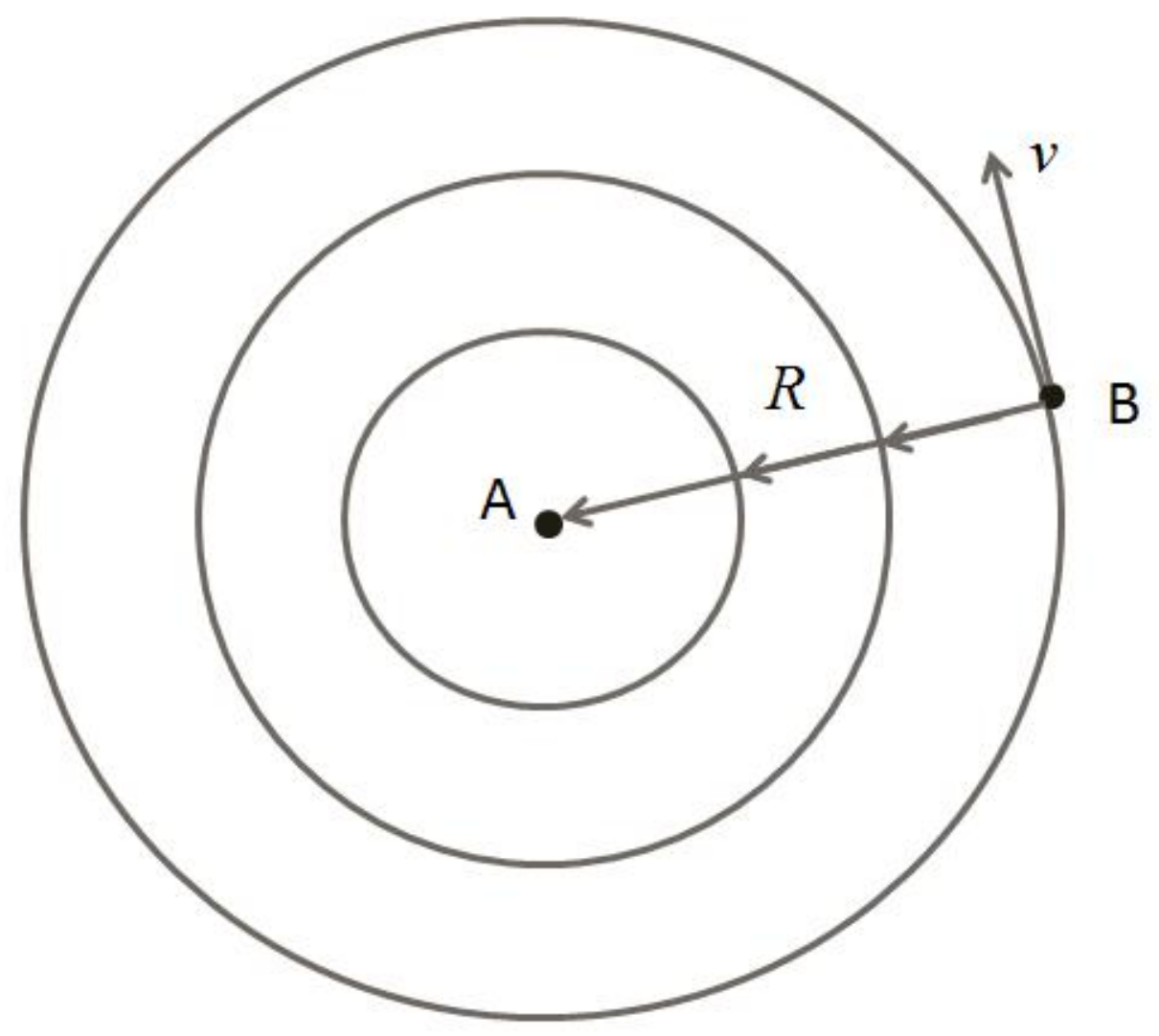

From A's point of view, the universal gravitation $\mathrm{F}$ of $\mathrm{A}$ on $\mathrm{B}$ is the elastic contraction force induced by $\mathrm{A}$ on $\mathrm{B}$ in the ether space, which is also the centripetal force $\mathrm{F} 1, \mathrm{~F}=\mathrm{F} 1=\left(\mathrm{v}^{2} / \mathrm{R}\right) \times \mathrm{M}_{\mathrm{B}}, \mathrm{v}^{2} / \mathrm{R}$ is the linear centribular acceleration caused by deformation in the ether space caused by $\mathrm{A}$ at the space position $\mathrm{R}$ 
away from $\mathrm{A}$ (that is, the position of $\mathrm{B}$ ), $\mathrm{M}_{\mathrm{B}}$ is the mass of $\mathrm{B}$. From the universal gravitation formula $\mathrm{F}=\mathrm{GM}_{\mathrm{A}} \mathrm{M}_{\mathrm{B}} / \mathrm{R}^{2}$; the centripetal force formula $F 1=\left(V^{2} / R\right) \times M_{B}$, because $F=F 1, \quad$ Plug into the equation: $G=\left(V^{2} / R\right) \times\left(R^{2} / M_{A}\right)$, the physical units are : $\left(\mathrm{v}^{2} / \mathrm{m}\right) \times\left(\mathrm{m}^{2} / \mathrm{kg}\right)$, the same physical units as $\mathrm{G}$ in Newton's formula for universal gravitation.

The following conclusions can be deduced from the formula $G=\left(V^{2} / R\right) \times\left(R^{2} / M_{A}\right):$

(1) $V^{2} / \mathrm{R}$ is the linear acceleration caused by the mass object $A$ at B's position in the etheric space. B is R away from the barycenter of $\mathrm{A}$, and the acceleration direction is from $\mathrm{B}$ to the barycenter of $\mathrm{A}$.

(2) $M_{A}$ is the mass of $A,\left(V^{2} / R\right) / M_{A}$ is the linear acceleration at B's position caused by the per unit mass in the etheric space. $\mathrm{B}$ is $\mathrm{R}$ away from the barycenter of $\mathrm{A}$, and the acceleration direction is from $\mathrm{B}$ to the barycenter of $\mathrm{A}$.

(3) The way in which an object per unit mass causes elastic deformation in etheric space is that: with the center of gravity of A as the center, it diffuses uniformly around in spherical shape. Therefore, the linear centripetal acceleration $\left(\mathrm{V}^{2} / \mathrm{R}\right) / \mathrm{M}_{\mathrm{A}}$ generated is the same at all positions of the spherical shell surface with A barycenter as the center and A radius of $\mathrm{R}$ (the spherical shell area is $4 \pi \mathrm{R}^{2}$ ), therefore, the acceleration of the elastic contraction of the whole spherical shell towards the center of the sphere should be $\left(V^{2} / R\right)(4 \pi$ $\left.\mathrm{R}^{2} / \mathrm{M}_{\mathrm{A}}\right)$

(4) We know, $G=\left(V^{2} / R\right) \times\left(R^{2} / M_{A}\right)$ is the gravitational constant, because $4 \pi$ is 
also a constant, therefore, the acceleration of the elastic contraction from the corresponding spherical shell surface to the center point of the sphere caused by the deformation in etheric space is also constant. $\quad\left(V^{2} / R\right)\left(4 \pi R^{2} / M_{A}\right)=a_{S}(a$ is the acceleration, and $\mathrm{S}$ is the shell area).

\subsubsection{Analysis and verification from the perspective of shrinkage of ether} space.

(1) $a_{S} \times M_{A}$ is the acceleration of the elastic contraction of the spherical shell surface caused by A mass object A with mass $\mathrm{M}_{\mathrm{A}}$;

(2) $\mathrm{a}_{\mathrm{S}} \times \mathrm{M}_{\mathrm{A}} / 4 \pi \mathrm{R}^{2}$ is the linear acceleration caused by the mass object A at B's position in the etheric space. B is R away from the barycenter of $\mathrm{A}$, and the acceleration direction is from $\mathrm{B}$ to the barycenter of $\mathrm{A}$.

(3) $\left(a_{S} \times M_{A} / 4 \pi R^{2}\right) \times M_{B}$ is the centripetal force $F 1$ induced by A of mass B.

(4) will be $a_{S}=\left(V^{2} / R\right)\left(4 \pi R^{2} / M_{A}\right)$, and $G=\left(V^{2} / R\right) \times\left(R^{2} / M_{A}\right)$ into $F 1=a_{S} x$ $M_{A} M_{B} / 4 \pi R^{2}$, it is concluded that $F 1=G_{A} M_{B} / R^{2}$. Because the centripetal force exerted by mass A on mass B in the ether is universal gravitation, so F1 = $\mathrm{GM}_{\mathrm{A}} \mathrm{M}_{\mathrm{B}} / \mathrm{R}^{2}=\mathrm{F}$.

\subsection{Conclusion of gravitational constant $G$}

From the above derivation, If ether exists as a space background medium and has the physical properties set above, the physical meaning of the universal gravitational constant $\mathrm{G}$ is as follows:

Mass objects in etheric space will cause elastic deformation in etheric space, which will spread evenly from the center of gravity of mass objects to the 
surrounding space; With the continuous expansion of the sphere formed by deformation diffusion in ether space, the elastic contraction force from the center of gravity of the mass object (also the center of the sphere) is exerted on all positions of the corresponding spherical shell surface, all spherical shell surfaces produce centripetal acceleration towards the barycenter of mass, and the acceleration of elastic contraction of spherical shell surfaces is $\left(V^{2} / R\right)(4 \pi$ $\left.\mathrm{R}^{2} / \mathrm{M}_{\mathrm{A}}\right)=4 \pi \mathrm{G}$. Therefore, the physical significance of the universal gravitational constant $G$ should be the elastic recovery coefficient of the curvature in ether space. In this way, the universal gravitational constant $G$ makes the etheric space assume mechanical properties and has utility, so the existence of etheric space is completely possible.

\section{Conjecture of macroscopic and microscopic physical properties of}

\section{ether}

Although we can not directly feel the ether, but through the use of ancient Greek philosophers, modern physicists to the concept of the characteristics of the too, and based on the Hubble red shift nature, universal gravitation constant physical significance analysis, we can boldly guess the mysterious fifth element - the attribute of the ether.

\subsection{Macroscopic physical properties of ether}

As the macroscopic background medium of the universe, ether should have the following macroscopic physical properties:

(1) The ether is filled with universal space (called etheric space), otherwise 
there would be no carrier for everything to exist and move, and gravitation is a function that transcends distance.

(2) The ether is transparent, otherwise we wouldn't be able to see light.

(3) The etheric space is negative energy, which can neutralize the positive energy radiated by photons, otherwise the universe must be bright everywhere because it is full of photons, there is no darkness.

(4) The density (negative energy/volume) distribution in the etheric space is uniform overall, but not completely uniform locally, otherwise the universe would not show the observed isotropy and $3 \mathrm{~K}$ cosmic background radiation equilibrium.

(5) Etheric space is a fluid state, and the spatial density always tends to be distributed uniformly from non-uniform to uniform, that is, from high density space to low density space, it automatically tends to be balanced and uniform, otherwise etheric space does not have an elastic restorative force, namely gravity.

(6) Etheric space is a continuous whole, otherwise it is impossible to conduct the elastic restorative force, namely gravitation, throughout space.

\subsection{Microscopic physical properties of ether}

Reasoning about the microscopic physical properties of the ether can only be explored through thought experiments and in conjunction with the properties of photons. In my book "Hypothesis about the Motion of Photon in the Space" （Open Access Library Journal, DOI: 10.4236/oalib.1105907）, I put forward 
the following logical inferences about the attributes and nature of photons:

\subsubsection{Properties of photons.}

(1)A photon is a particle moving in a uniform spiral line in space. Therefore, the moving mass of a photon is a moving particle in ether space.

(2) The energy of a photon is the product of the particle's motion frequency and Planck's constant. Since the motion mass of a photon is determined by its energy, and the photon energy is $\geqslant 0$, therefore, the motion mass of a photon is $\geqslant 0$.

(3)A photon particle has a spatial extension. Given that the etheric space has extendedness, photons are particles moving in the etheric space, If there is no space extension of the photon particle, it is impossible for the photon to exist and move in the ether space, there is no connection between the photon particle and the ether space, and the universal gravitation will not affect the motion of the photon.

(4) The motion mass of the photon is determined by the motion frequency of the particle, so the motion mass of the photon has nothing to do with the extension of the particle in the ether space, so, the mass of the photon particle can be changed, but the space extension of the particle remains unchanged. The photon particle is the concentrated expression of its mass or positive energy and the scale of the photon particle space should be much smaller than the Planck length.

4.2.2 Microscopic properties of ether. Photons exist and move in the ether, 
and the two are connected. According to the essential properties of photon, it is speculated that ether should have the following microscopic properties:

(1) The ether is space itself. If the void exists and the ether is merely fleshed out into the void as a medium, then photon and ether must negate each other: In the void, there are no photons where there is an ether, and there are no ether where there are photons(A photon particle has a spatial extension). We know that a photon is a particle of mass, and if a photon moves in the void, the moving particle must constantly push away the ether in front of it, then there will always be resistance to the movement of the photon, which must continue to consume photon energy, that is, the photon should continue to radiate energy outward. However, according to Planck's quantum theory, the outward radiation energy of photons is discontinuous, which is in quantum state one by one. Therefore, the void hypothesis contradicts quantum theory, and the void cannot exist. Because there is no void, the ether is space itself, the ether is the extension of space. If the ether disappears, the corresponding space also disappears.

(2) The etheric space completely penetrates the photon particle. Photon is a particle moving in ether space, which is the concentrated expression of positive energy (or moving mass) and has space extension; knowing that the ether is negative energy and space itself, since the photon particle exists and moves in the ether space, and the relation between the particle and ether is the affirmation, the spatial extension of the particle must overlap completely with the ether space. 
(3) There is no resistance to the motion of a photon particle in etheric space. The photon moves at a constant speed in the etheric space. Since the energy radiated by the photon is discontinuous, it indicates that the photon energy is not continuously consumed. Therefore, there is no resistance to the movement of the photon particle in the etheric space.

(4) Dense etheric space exerts a force on the motion of the photon particle. The elastic contraction force in etheric space ( universal gravitation) is essentially the mass object affecting the uniform distribution of etheric space, resulting in the flow potential energy (similar to voltage difference) generated in dense etheric space relative to thin etheric space. Because the moving photon is affected by universal gravitation, the dense etheric space will exert a force on the movement of the photon particle.

(5) The etheric space absorbs the positive energy radiated by neutralized photons and its density decreases. The positive energy of galactic starlight radiation is absorbed and neutralized by the etheric space and annihilated, so the etheric space will inevitably lose the corresponding negative energy. Since there is no evidence that the etheric space is constantly shrinking, the density of etheric space must decrease, and the corresponding elastic contraction force of etheric space will decrease, the gravitational constant $\mathrm{G}$ will go down. Since the loss of starlight energy is proportional to time (see 2.2), the density of the etheric space decreases year by year, and the magnitude of the universal gravitational constant $\mathrm{G}$ is inversely proportional to the age of the universe, 
which also conforms to the Dirac Large number conjecture.

\subsubsection{Supplementary interpretation of the zero result of Michelson}

Morley's experiment. The first chapter puts forward a possible explanation for the zero result of Michelson Morley's experiment, that is, ether, as the background medium of space, has the property different from mass material, and does not interact mechanically with moving light under certain conditions, that is, the interaction force is zero. Since the ether is space itself, completely overlapping with the photon particle in the spatial extension (see 4.2.2), the ether has no resistance to moving photons. If the moving photon is considered to be stationary, the "etheric wind" moves at the speed of light $\mathrm{c}$ relative to the photon, but does not exert a force on the photon. Therefore, the "etheric wind", which is far less than the speed of light and equivalent to the speed of the earth's revolution $(30 \mathrm{~km} / \mathrm{s})$, does not exert a force on the photon, so the direction of the photon's movement does not change, and it is impossible to measure the existence of "etheric wind " in the experiment.

\section{Some conjectures about the significance of the ether and the photon}

\section{in making up the universe}

From the inference of the existence and physical properties of ether, and from the hypothesis of the nature of photon, ether and photon are opposites and unity of existence. It would certainly be very exciting to speculate further on the significance of the ether and photons to the formation of the entire universe.

\subsection{Etheric space is absolute space}


5.1.1 Definition of absolute space. The concept of absolute space, which exists independently of anything and is always the same and motionless, was put forward by Newton. Newton further defined the concepts of "absolute motion" and "absolute rest", and in 1689 proposed the famous bucket experiment, which proved the existence of absolute motion. But Einstein believed that space is relative, there is no absolute space. So does absolute space exist? In fact, the two great physicists' understanding of absolute space was not contradictory: according to Newton, the most important property of absolute space is that it is possible to determine whether a mass is in a state of absolute rest or absolute motion; Einstein's denial of absolute space is that mass can bend space, and since mass is moving, space itself is not a static state. As you can see, it's not an either-or issue.

According to Newton's and Einstein's viewpoints, absolute space refers to the absolute nature of the space-time coordinate system in this space. Relative to the coordinate system is moving objects, it is absolute motion, but the absolute space itself can expand and bend deformation.

5.1.2 Etheric space is absolute space. According to the Doppler effect, the earth is not absolutely static, and the space coordinate system established with the earth as the origin is definitely not absolutely static. Therefore, relative to the earth space coordinate system, the speed of light is not C. Similarly, the solar system is also moving, and the space coordinate system based on the sun is also moving, and the speed of light is not $\mathrm{C}$ with respect to the solar space 
coordinate system. According to inference 4.2.2, ether is space itself, and there is no void. Therefore, the speed $\mathrm{C}$ of light in vacuum is the speed of motion in ether space. It can be seen that the speed of light is relative to the etheric space coordinate system. Since the speed of light $\mathrm{C}$ is constant, the etheric space coordinate system relative to the constant speed of light must be absolutely static. According to the Big Bang theory, the universe was formed by an extremely small singularity through the Big Bang. After the Big Bang, space, time and light were generated. Since the cosmic singularity was absolutely static before the Big Bang (because there could be no space), if the cosmic singularity was established as the origin of the space coordinate system, we would find that the light generated after the Big Bang in this space coordinate system, the distance divided by the movement time is constant $\mathrm{C}$. Therefore, the space coordinate system established with the cosmic singularity as the origin must be absolutely static, and the ether space, which is constant relative to the speed of light, is absolute space.

If it is found that a fundamental particle in the etheric space is not affected by the photon's Doppler effect (that is, the frequency and wavelength of the photon are not changed after the collision), it can be said that the fundamental particle is absolutely static in the etheric space.

\subsection{Photon and Etheric vortex form elementary particles}

All elementary particles such as electrons, protons, neutrons, etc., are formed by the fusion of photons and ether. 
5.2.1 Formation of elementary particles. According to the photon hypothesis, a photon is a particle moving uniformly in a spiral line in space. Although the etheric space is uniformly distributed as a whole, there are locally dense etheric spaces. As we know, there is no resistance for the photon to move in the uniform etheric space, but when the photon moves to the dense etheric space, the linear motion of the photon is blocked by the dense ether, so that the linear motion of the photon is transformed into circular motion. At the same time, the photon captures a portion of the ether and spins it to form a sphere. The photon particle moves along the surface of the sphere in an S shape, so that the photon merges with the ether and the elementary particle is formed (see image below) . 


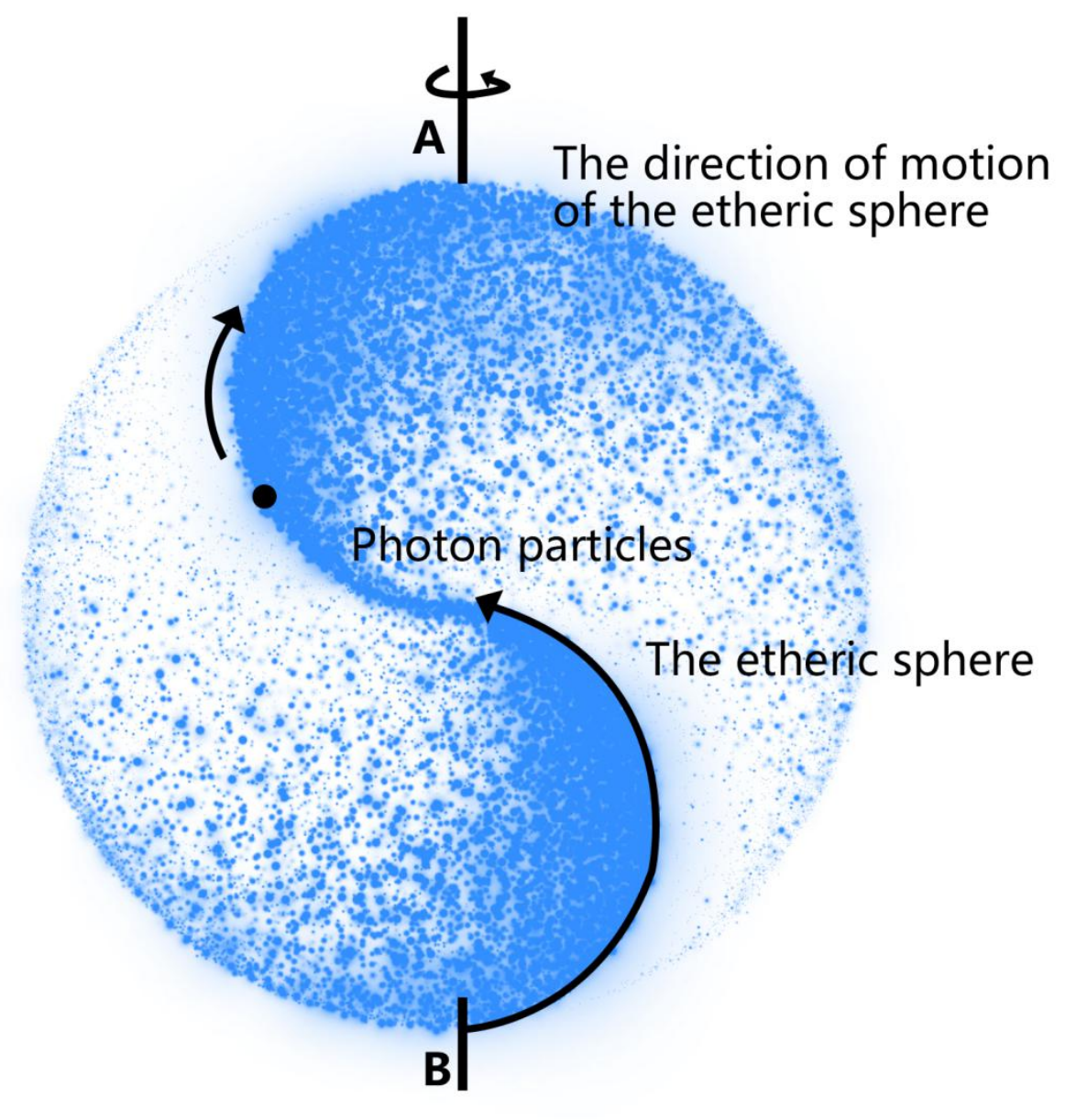

$B \rightarrow A$ The path of motion of the photon particle

The figure shows $\mathrm{A}$ fundamental particle. $\mathrm{B} \rightarrow \mathrm{A}$ is the track of the photon particle moving along the surface of the etheric sphere, which is A closed S-shaped curve. The Linear Velocity of a photon particle moving along a curve on the surface of an etheric sphere is $\sqrt{2} \mathrm{C}$, a point on the surface of a spherical shell perpendicular to the axis of rotation and at a distance of $\mathrm{R}$ from the center of the sphere, the line velocity of rotation is also C.

The properties of elementary particles are as follows. 1 photon fuses 1 etheric sphere (sphere radius $\mathrm{R}$ ) to form 1 elementary particle, the photon particle makes s-type closed motion on the surface of the etheric sphere, which 
is the combined motion of two circular motions perpendicular to each other, the linear velocity of the resultant motion $\sqrt{2} \mathrm{C}$; the linear velocity of the photon particle moving along the two circumferential planes is $\mathrm{C}$. The photons in the elementary particle are positive energy, and the etheric sphere is negative energy. Affected by the circular motion of photons, the etheric sphere rotates continuously like the earth; the point on the surface of the etheric sphere is perpendicular to the spin axis and $\mathrm{R}$ away from the center of the sphere, and its linear velocity is also $\mathrm{C}$. The positive energy of the photon in the elementary particle is always equal to the negative energy of the etheric sphere; as the photon positive energy in the etheric sphere changes, so does the etheric sphere negative energy. The larger the movement frequency of the photon particle, the larger the mass of the elementary particle, the smaller the volume of the etheric sphere, and the higher the negative energy density of the sphere; on the contrary, the smaller the mass of the elementary particle, the larger the volume of the etheric sphere, the lower the negative energy density of the sphere.

5.2.2 Etheric sphere of elementary particles. The etheric vortex formed etheric sphere through continuous rotation, which made the boundary between the elementary particles and the etheric space, and the elementary particles also formed self category. The phenomenon of ice in water is somewhat similar. There is a boundary between ice and water, but the ice and water are relationship of exclusion in space, and the elementary particle is coincident relation. As we know, the etheric space is a continuous whole, and the dense 
etheric space is formed by the superposition of the etheric negative energy. Therefore, the etheric sphere, which constitutes the fundamental particles, is completely overlapped with the etheric space. The etheric sphere of elementary particles is formed by photons capturing the dense etheric space, the etheric sphere and etheric space are completely coincident, so the etheric sphere rotation will not drive the corresponding etheric space rotation.

5.2.3 Collision of elementary particles. Since the space extension of the photon particle in the elementary particle is much smaller than the Planck length, the collision of one photon with one elementary particle cannot be a collision of the photon with the photon particle in the elementary particle, but a collision of the photon with the etheric sphere of elementary particle. In the same way, the collisions between fundamental particles such as electrons and protons are also collisions between their etheric spheres. Collisions between elementary particles and photons, or between elementary particles, produce energy exchange. For example, a photon collides with an electron, and if the energy of the photon increases and the energy of the electron decreases after the collision, the frequency of the moving particle in the electron elementary particle decreases, at the same time, a part of the negative energy of the electronic etheric sphere is released into the space, so that the negative energy of the electronic etheric sphere is always equal to the positive energy of the electronic particle; if the energy of the electron increases after the collision, the frequency of the moving particle in the electron elementary particle increases, and the electron etheric 
sphere captures some of the negative energy from etheric space, the negative energy of the electron etheric sphere is always equal to the positive energy of the particle.

5.2.4 Space motion of elementary particles. It can be known from 4.2.2 that there is no resistance to photon movement in ether space; It is also known that the etheric sphere of elementary particles completely overlaps with the etheric space, and the rotation of the etheric sphere will not drive the rotation of the corresponding etheric space that overlaps with it, since elementary particles are composed of photons and etheric spheres, there is no resistance to the movement of elementary particles (less than the speed of light) in etheric space.

5.2.5 Elementary particles have rest masses. As can be seen from 5.2.1, the photon particle of the elementary particle keeps s-type closed-loop rotation along the surface of the etheric sphere, and the etheric sphere of the elementary particle also keeps rotation at the same time, but the spatial coordinates of the etheric sphere of the elementary particle can remain static relative to the etheric coordinate system. According to 5.1, the ethereal space is absolute space. If the elementary particle is at rest with respect to the ethereal space coordinate system, the elementary particle is at absolute rest, but this does not prevent the motion of the photon particle and the ethereal sphere. If the positive energy in the absolute rest elementary particle is $\mathrm{E}$ (negative energy equals positive energy), then its rest mass is $\mathrm{m}=\mathrm{E} / \mathrm{C}^{2}$. Because the photon always moves in the space, can not relative to the space coordinate system at rest, so the photon 
has no rest mass, only the motion mass.

\subsection{The formation of electrical properties of elementary particles}

The different combination of the spin direction of the ethereal sphere and the S-type closed-loop motion of the photon particle causes the elementary particle to be positively charged, negatively charged and negatively charged.

5.3.1 Charged elementary particles. The spin direction of the etheric sphere is perpendicular to the S-type closed-loop Motion Direction of the Photon Particle (in the case of right-handed and left-handed rules) . The motion of the photon particle cuts the etheric sphere' s spin direction, creating a positive or negative charge in the elementary particle (see figure below) . A elementary particle has a basic charge of 1 unit, which is $1.602189 \times 10^{-19}$ coulombs.

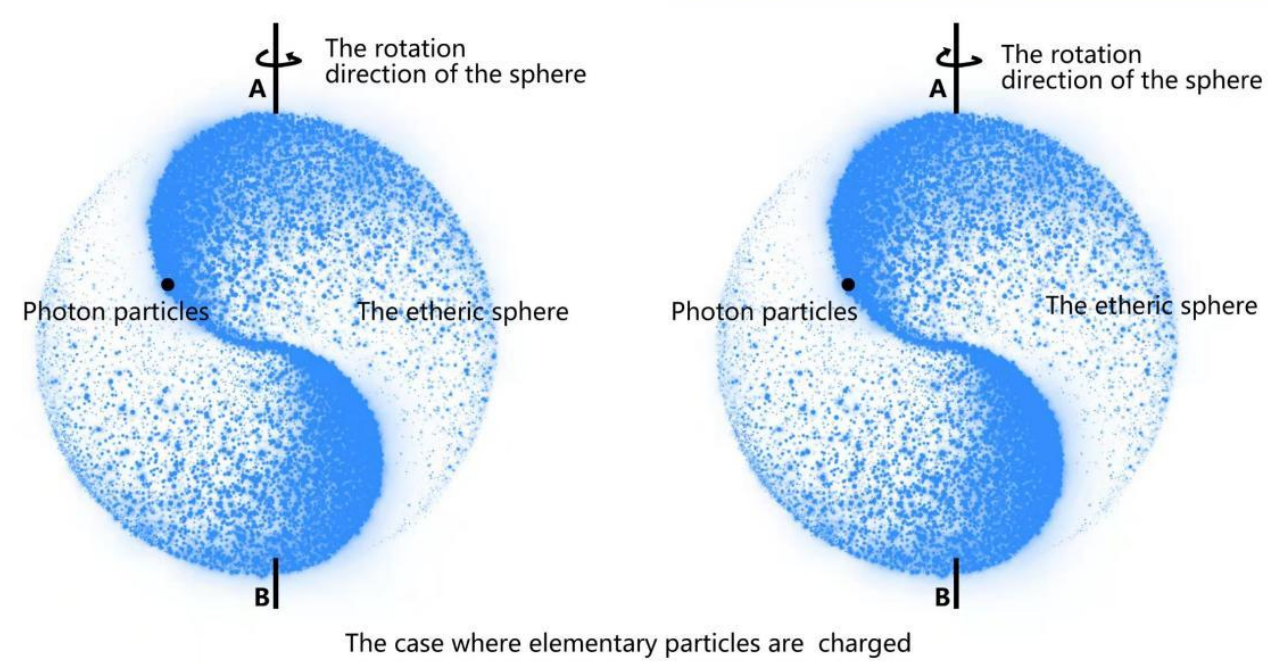

5.3.2 Elementary particles are not charged. The rotation direction of the 
etheric sphere is parallel to the direction of the S-type closed-loop motion of the photon particle (in the same or opposite directions). The motion of the photon particle can not cut the rotation direction of the etheric sphere, so no electric charge is produced, such elementary particle are uncharged (see image below) .

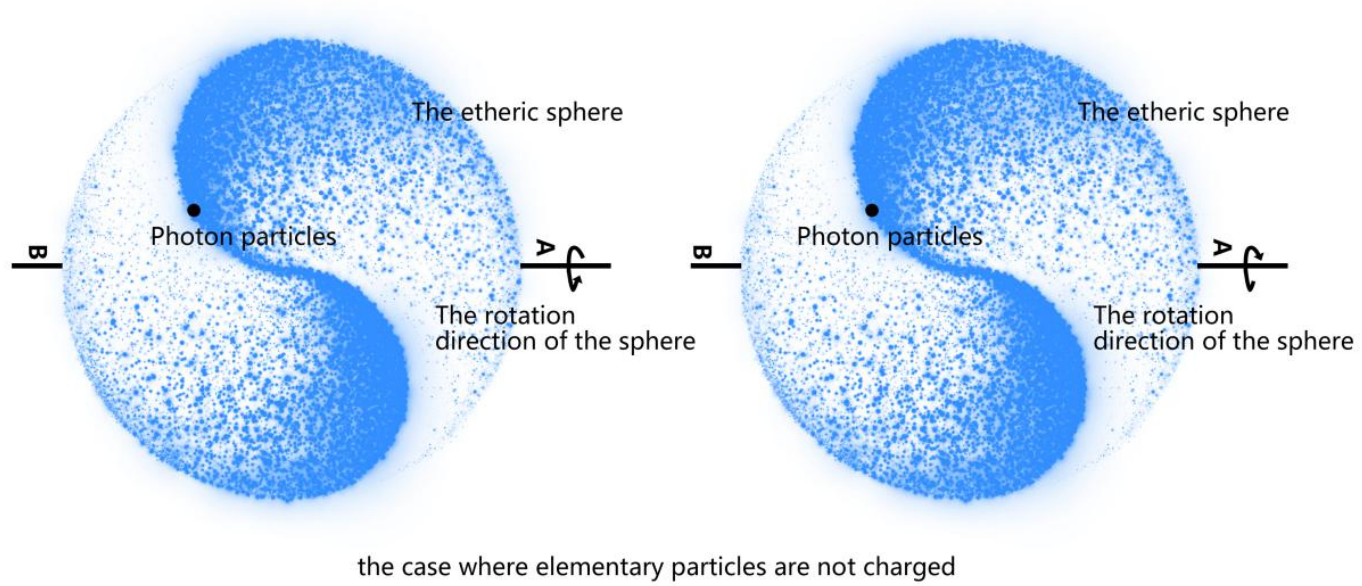

\subsection{Explanation of fundamental forces}

Universal gravitation, strong force, weak force and electromagnetic force are four basic forces in nature, which have not been unified theoretically in the physical world. Gravity has been explained from the point of view of the elastic contraction force of the ether, the other three forces continue to try to understand from the point of view of the ether, the specific formula and calculation is still to be proved by physicists.

5.4.1 Strong force. Between the Nuclei of an atom, there exists a strong interaction force which overcomes the coulomb repulsion-- strong force. At the 
atomic scale, the strong force is much larger than the Coulomb force; but strong force is a short-range force that falls quickly when nucleons are too far apart. From the ether' $\mathrm{s}$ point of view, strong force is due to the binding force produced by the partial coincidence of the proton and the neutron' $\mathrm{s}$ ether sphere. When the etheric spheres of protons and neutrons come into contact with each other, the two etheric spheres first generate resistance; when they are further squeezed, parts of the etheric spheres overlap and bond with each other, that holds protons and neutrons together. Because strong force is generated through the contact of etheric spheres of protons and neutrons, it is a short-range force. If you go beyond the diameter of the etheric sphere, no strong force will be generated without contact between the two etheric spheres.

5.4.2 Weak force. Weak forces also exist between elementary particle forces, acting at much shorter distances and much less intense forces than strong forces. The weak force exists only at the moment of the decay and trapping of the particle, and disappears as soon as the decay and trapping process is over. For example, a neutron in the nucleus of an atom emits one electron and one neutrino as it $\beta$ decays into a proton, this is the action of the weak force. Similarly, from the perspective of the ether, neutron decay into a proton is a process in which the neutron releases part of its positive and negative energy (electrons, neutrinos). From 2.2, it can be seen that the external radiation energy of photons in space is neutralized with the negative energy in space, but the elementary particle is different. Because of the spin of the etheric sphere, the 
photon particle of the neutron radiated two photon positive energy discontinuously, which combined with the negative energy of the etheric sphere to form electron and neutrino ejected from the neutron; at the same time, the frequency of the photon particle decreases, and the s type closed loop motion of the photon particle changes from parallel to perpendicular to the rotation direction of the etheric sphere, and the elementary particle produces a positive charge, which is transformed into a proton. The process by which protons decay into neutrons is the process by which protons trap positive and negative energies (electrons, neutrinos) : after protons capture electrons and neutrinos, the positive energy of protons absorbs the positive energy of electrons and neutrinos to form a photon particle, and the motion frequency increases accordingly, the particle $\mathrm{S}$ closed-loop motion direction changes from perpendicular to the spin direction of the ethereal sphere to parallel, the elementary particle is not charged, and is converted into neutrons; at the same time, the electron and neutrino etheric spheres fuse into the proton' s etheric spheres.

As shown in 5.2.3, the force between the elementary particle is transmitted through an etheric sphere, so in the course of $\quad \beta$ decay, as the elementary particle ejects or captures electrons and neutrinos, the force that the etheric sphere of the elementary particle will produce in an instant when it throws out or combines negative energy is called the weak force.

5.4.3 Electromagnetic force. Electromagnetic force arises from electrostatic force. The electrostatic force is the interaction force between two static point 
charges Q 1 and Q 2 in a vacuum. Its magnitude is proportional to the product of Q 1 and Q 2 and inversely proportional to the square of the distance R between them; like charges repel, unlike charges attract. As shown in 5.3.1, the S-type closed-loop motion of the photon particle vertically cuts the spinning etheric sphere, making the elementary particle positively or negatively charged. From 5.2.2, we know that the spin of the elementary particle will not push the spin of the etheric space that coincides with it; however, the rotation of the charged elementary particle of the etheric sphere causes vibrations the etheric space that overlaps with it, which are transmitted to the surrounding space in the form of "Faraday' s force lines" (referred to as "vibrational force lines" for short); the "vibrating force line" of the charged elementary particle is uniformly spherical in shape from the charge center to the surrounding space; the farther the vibration conduction distance $\mathrm{R}$ is from the charge center, the weaker vibration frequency $\mathrm{F}$ is. When the vibration conduction distance from the charge center is $\mathrm{R}$, the product of the vibration frequency $\mathrm{F}$ at the position $\mathrm{R}$ and the sphere area $4 \pi R^{2}$ is the electrostatic constant $K$. The same kind of charge produces the "vibration force line" in the same direction, but the different kind of charge in the opposite direction. The S type closed loop motion of the uncharged elementary particle particle is parallel to the spin motion of the etheric sphere, thus shielding the effect to the etheric space from the spin motion of the etheric sphere, no "Faradite force line" vibration waves are generated to the surrounding space. 
Opposite charges "vibration force line"in the opposite direction, and the two waves cancel each other out, causing the two dissimilar charges to attract each other (see figure below) .

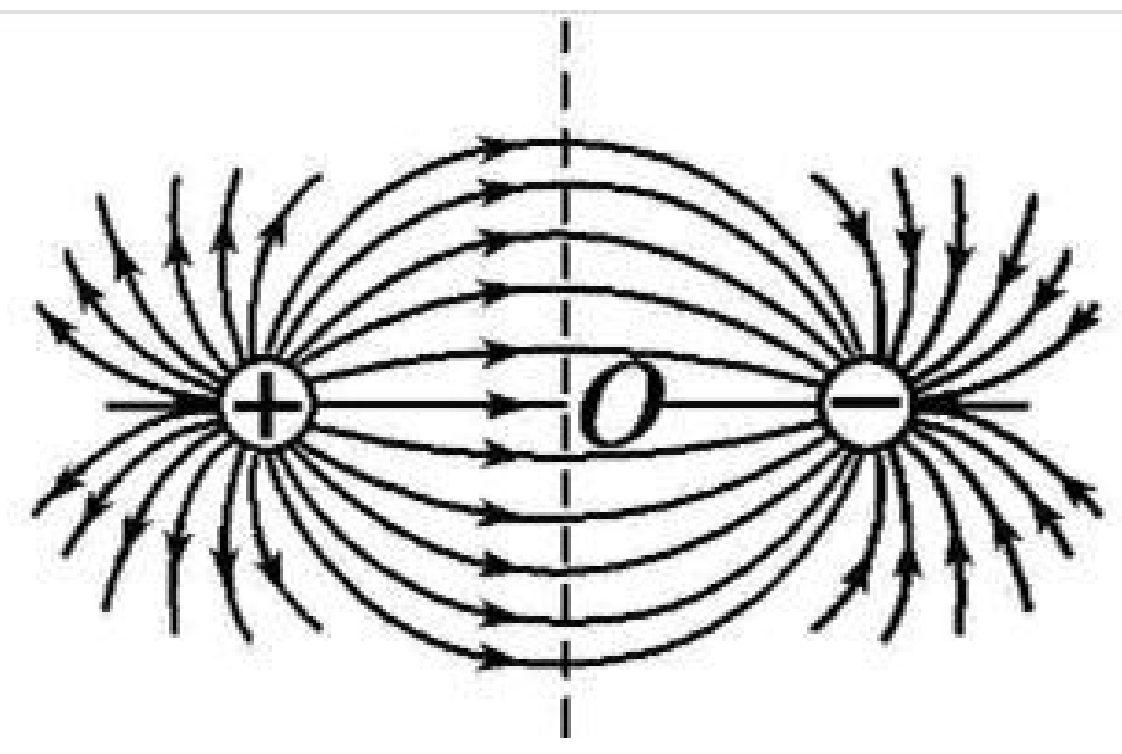

The same charge "vibration force line"in the same direction, two waves of vibration superimposed each other, resulting in two charges of the same species repel each other (see below) .

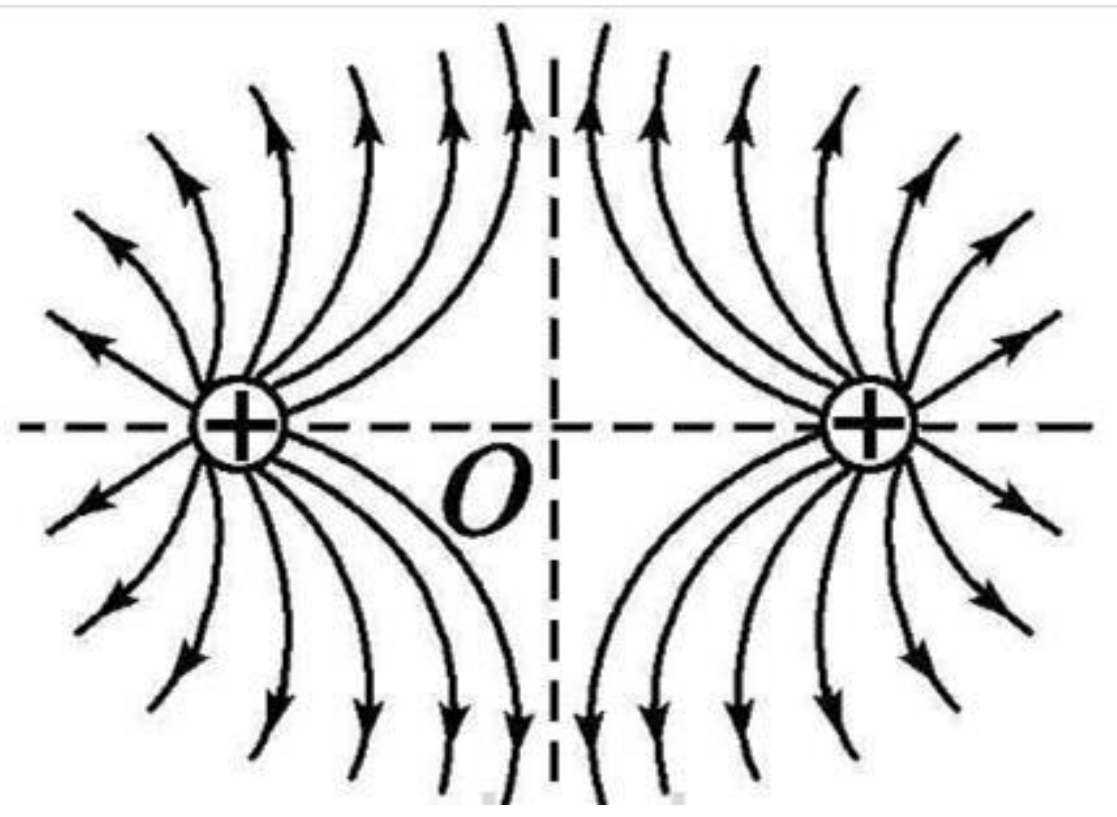




\subsection{Elementary particles make up atoms}

As can be seen from 5.2.1 formation of elementary particles and 5.4 formation of fundamental forces, Protons, neutrons and electrons are all rotating etheric spheres, and their photon particles move in an S-shaped closed loop along the surface of the etheric sphere; also known as 5.2.2, the ether spheres can overlap, so presumably the atoms should be made as follows: because the electron mass is small, the electron etheric sphere is larger than the Proton etheric sphere, and the density of the etheric sphere is lower than that of the Proton etheric sphere; the S-type closed-loop motion radius of the photon particle of the electron is larger than that of the proton, which is equivalent to the S-type closed-loop motion of the electron around the proton. The etheric spheres of protons and the etheric spheres of electrons overlap to create a strong force that stabilizes the hydrogen atom, so there's no need to worry about electrons falling onto protons (see photo below). Due to the small density of the electron-etheric sphere, the binding force between the electron and the proton is smaller than that between the proton and the neutron. 


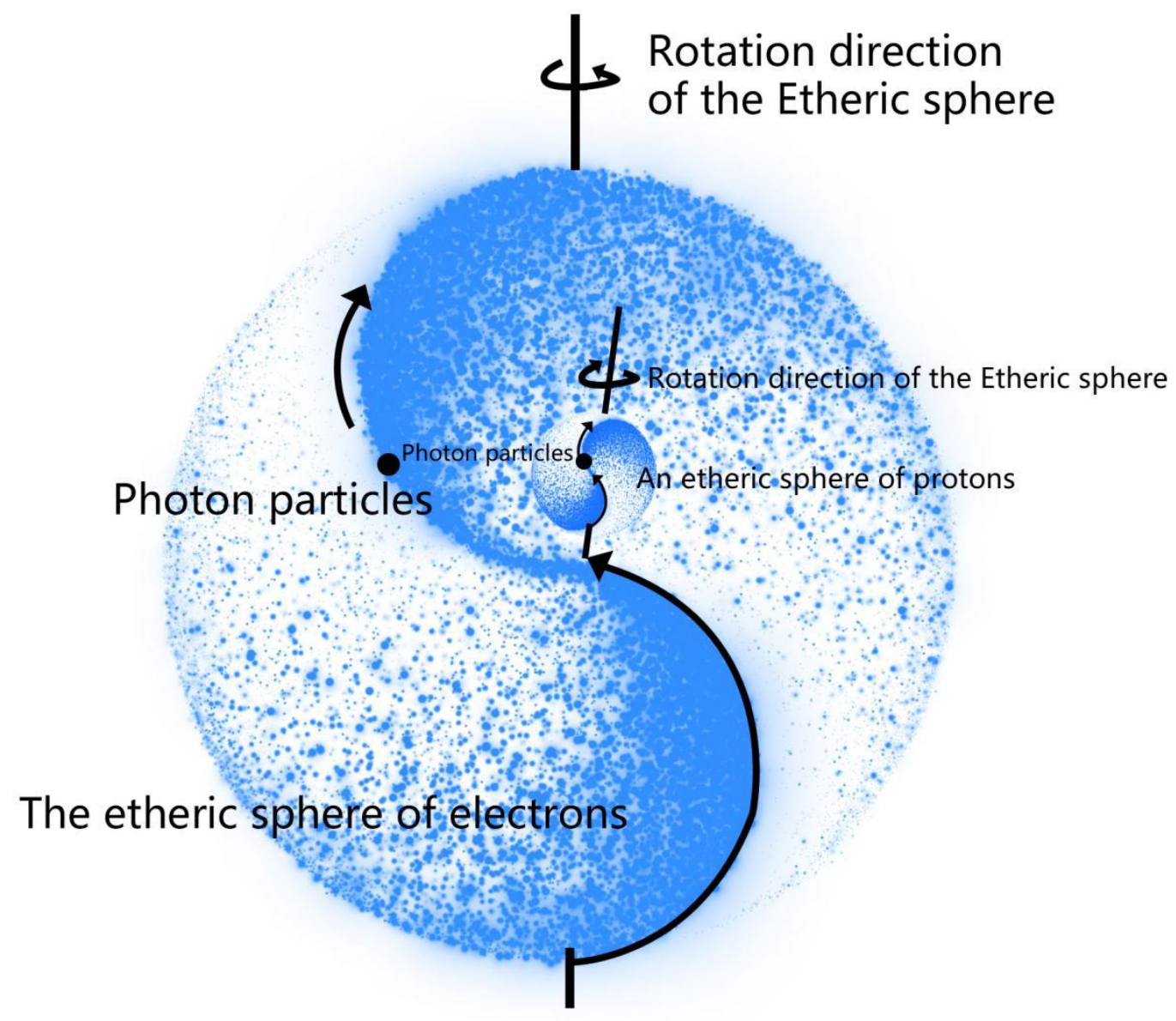

Hydrogen structure diagram

Atoms of many protons, neutrons and electrons are also made on this principle (see diagram below) . Etheric spheres of protons and neutrons overlap to form nuclei; an etheric sphere of multiple electrons overlaps with the nucleus to form atoms. In an atom: the electron etheric sphere with small mass (positive energy) has a large volume and a small negative energy density. The photon particle moves on the surface of the etheric sphere in an S-type closed motion, and its orbit is far from the nucleus; the electron etheric sphere with large mass (positive energy) has small volume and high negative energy density. The 
photon particle moves in S-type closed motion on the etheric sphere surface, and its orbit is close to the nucleus.

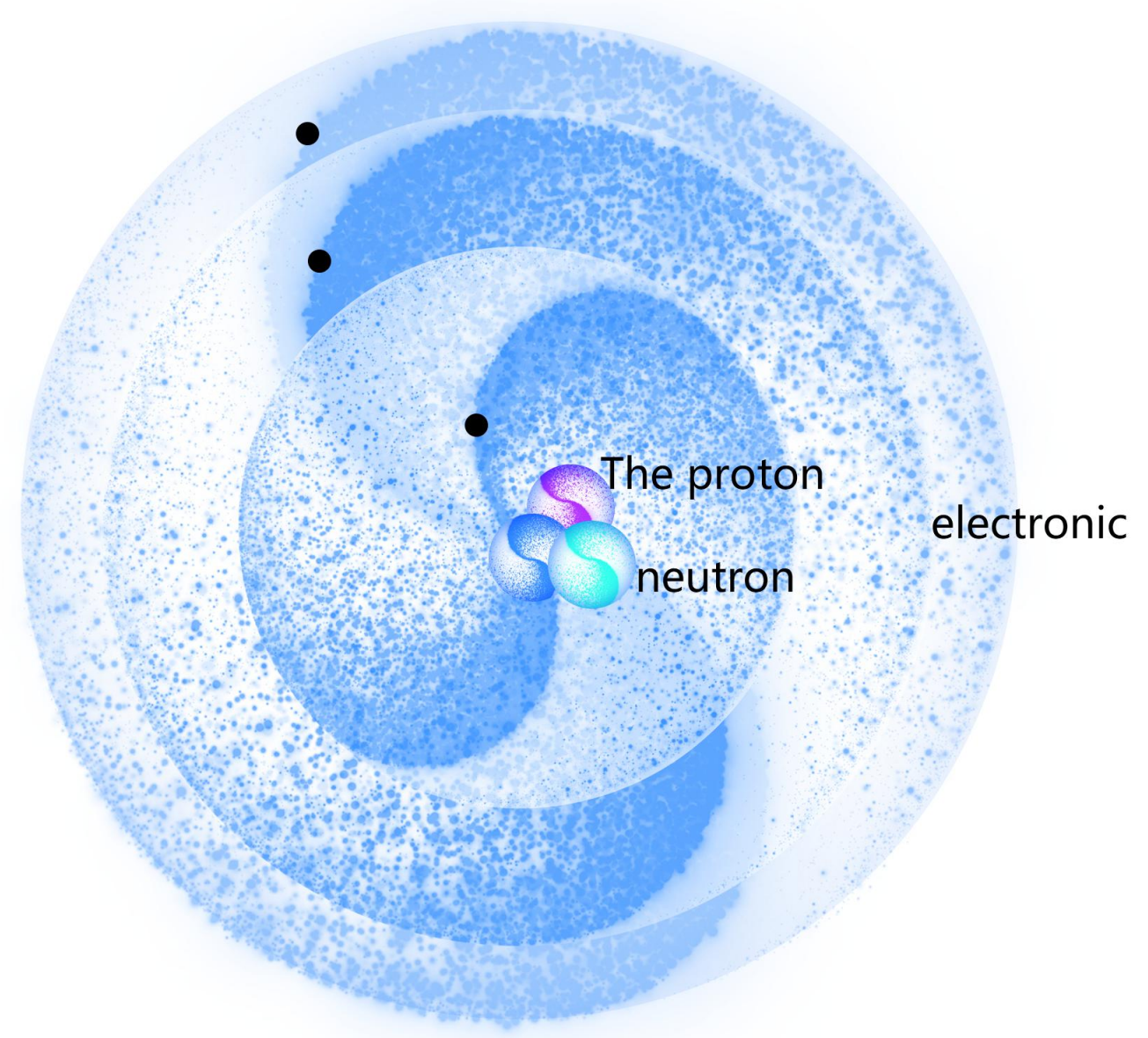

\section{An atom composed of multiple protons, neutrons and electrons}

\section{Ether is the stream of consciousness hypothesis}

We can feel that consciousness exists as much as matter, but there is still no definite definition of what consciousness is. How consciousness relates to matter has also been a headache for philosophers to this day. The Great Leibnitz put forward the famous "Monad Theory", that monads have both the properties 
of matter and consciousness. Inspired by this, and combined with the imagination of ancient Greek philosophers on the ether, the bold inference: If the concept of matter is abstracted into positive energy or mass (including the rest mass and the moving mass), then matter is moving photon and the moving particle in the elementary particle. Consciousness corresponding to matter is therefore negative energy or non-mass, so consciousness must be ether. Etheric space is the consciousness of the universe. It permeates all things in the universe, so it can sense, remember and influence the movement of matter. Elementary particles (except photons moving in space) are the unity of matter (positive energy or mass) and consciousness (negative energy or ether). The movement of photon particles of elementary particles forms mass. The etheric sphere spins to form elementary particle space categories and self-consciousness. Although the photons in space do not combine with the etheric sphere to form a elementary particle, they are always moving in etheric space and are therefore unified with the ether. Mass, the representative of matter, and ether, the representative of consciousness, are interdependent and mutually verified. The birth and death of either side will inevitably lead to the birth and death of the other side. Humans, too, are made up of elementary particle, and our consciousness is essentially a superposition of elementary particle self consciousness, gradually forming higher levels of consciousness, thought, and so on. In this sense, everything in the world is conscious, only at different levels, and the highest level of 
consciousness should be the universal consciousness, that is the etheric space, which contains everything, senses everything, remembers everything.

\section{The birth and death of the universe}

The most famous theories about the formation of the universe are the Big Bang theory, the steady state theory and so on. Based on the above deduction about the ether, guess the universe' $\mathrm{s}$ birth and death.

\subsection{The universe was created by design.}

7.1.1 The supreme logical law of Birth and death. If light is not created, light has no end, which is an axiom, so its inverse negative proposition proposition is also an axiom. According to 2.3, all light in space has an end, so light is be created. If all light ends, then the space that accompanies it must also end, and the universe will be nothing. The universe was created, because it will end. There is no beginning without an end, and there is an end must be with a beginning. This is truth beyond the universe.

\subsubsection{The laws governing the motion of all things in the universe are not}

in the universe. The constant speed of light $\mathrm{c}$, the universal gravitational constant G, Planck's constant h, how the ether and photons form elementary particles, how consciousness is generated, etc, are all expressed in the universe, but the laws that govern these constants and laws are not in the universe. In particular, absolute time (not measured time represented by any timing device, which is relative) enforces the existence and continuity of all things in the 
universe (including the ether space itself). It existed before the universe came into being. It still exists after the end of the universe. Therefore, the fundamental laws governing the existence and operation of the universe can only be caused by the external causes of the universe.

7.1.3 The universe was created from nothing. Because the universe has a beginning and an end, there must be no beginning, no end outside the universe, eternal existence of nothingness. So the universe can only be created from nothing.

\section{2 the beginning of the universe is a tiny seed}

The seeds of the universe are created from nothing with the same positive and negative energies.

7.2.1 Out of thin air. According to 5.2.1, the larger the mass or positive energy of the elementary particle is, the higher the frequency of its moving particle is, the smaller the volume of the etheric sphere is, and the higher the density of the etheric sphere is. Therefore, it is speculated that the universe was created out of nothingness as an extremely small sphere with a diameter much smaller than the Planck length, which is called the primordial etheric sphere. The primordial etheric sphere is a very high density ethereal sphere, there is a primordial photon particle moving in an S shape close-loop along the surface of the sphere; the linear velocity of the photon particle is much higher than that of $\sqrt{2} \mathrm{C}$, and the motion frequency is also very high. The positive energy of the 
primordial photon particle of the cosmic seed is always equal to the negative energy of the primordial etheric sphere. The mass of the cosmic seed is not less than the total mass of the current universe; the negative energy of the cosmic seed is no less than the sum of the current negative energy in the etheric space. Or you can think of the cosmic seed as the singularity of the Big Bang.

7.2.2 The Big Bang. Because the seeds of the universe contain so much positive and negative energy, the Big Bang of the seeds of the universe should have been completed in a flash, far less than Planck time, instantly creating a universe on a grand scale. The primordial etheric sphere formed the cosmic etheric space, and the negative energy density of etheric space decreased greatly than that of the primordial etheric sphere, presenting a balanced distribution on the whole, but with local fluctuations; The primordial photon particle of the cosmic seed is divided into numerous photons, which move in a uniform spiral straight line in the ether space, the velocity along the straight line is $\mathrm{C}$, and the linear velocity of the photon is $\sqrt{2} \mathrm{C}$.

7.2.3 The steady state universe. According to 2.2 , if the stars from all galaxies systemic redshift phenomenon is not caused by the doppler effect, but because of the photon energy decay over time lead to, then can be speculated that the expansion of the universe is not necessarily, it is likely to be still, but that doesn't deny that the universe could have had a big bang and then gone into a steady state. It can be speculated that after the big bang, the seeds of the 
universe entered the macroscopic and large scale stable state space immediately, the space extension remained unchanged and no longer expanded or contracted, but the density of the ether space gradually decreased with the age of the universe.

After the Big Bang, the primordial photons of the seeds of the universe split into countless photons moving in the etheric space. Some photons met the dense etheric space and combined to form elementary particles, which then combined into atoms and various elements, and gradually formed various substances, animals and plants and even human beings with high-level consciousness from low to high; and some photons continue to move, collide, and move around in space alone. It can be inferred from 2.2 that photons discontinuously radiate energy to the ether space and neutralize part of the negative energy in the ether space. In this way, the negative energy density of the ether space gradually decreases over time under the premise of wide extension and invariance, and finally disappears. As the density of etheric space thins as the universe ages, so should the gravitational constant.

\subsection{The end of the universe}

Since the decay of photon energy over time is quantized, that is, one photon after another radiates energy into space discontinuously, the energy of each photon moving in space will eventually decrease to 0 , and all photons will eventually die out. If you change formula 3 in 2.2 , you get the following result: 
$\triangle \mathrm{E} / \mathrm{T} / \mathrm{E}=\mathrm{kc}$ ( formula 4),$\triangle \mathrm{E}$ represents the energy lost by starlight in the time period $\mathrm{T}$ from the galaxy to our observation point; $\triangle \mathrm{E} / \mathrm{T}$ is the average energy loss coefficient of light in the process of motion, represented by $\mathrm{P}$, that is, $\mathrm{P}=\triangle \mathrm{E} / \mathrm{T}$; substituting $\mathrm{P}$ into formula 4 gives $\mathrm{P} / \mathrm{E}=\mathrm{kc}$ (Formula 5); and if I switch both sides of equation 5 , I get $\mathrm{E} / \mathrm{P}=1 / \mathrm{kc}$ (equation 6), the physical implication seems to be that all photons moving in space, at the average energy consumption rate of $\mathrm{P}$, are $1 / \mathrm{kc}$ away from the end of their lives, which means that all light is dead by then. When all photons die (including those of elementary particles), then the ether disappears and our universe ends. The universe goes from birth to death, ending in nothingness.

JinJjunCheng Chief Economist, development and Reform Commission of Anhui Province Email address: cdcdcd999@126.com;

DianCheng Senior Manager of State Grid Yingda International Trust Company Email address: 1226195145@qq.com

References:

1. Mathematical Principles of Natural Philosophy

2. Special relativity; General Relativity

3. A Brief History of Time

4. Monad Theory

5. Hypothesis about the Motion of Photon in the Space ( Open Access 
Library Journal, DOI: 10.4236/oalib.1105907） 